\title{
The logic structure deriving Lorentz transformation
}

\author{
${ }^{1}$ Chang-Wei Hu, ${ }^{2}$ Zhi-Heng Shi
}

1. Beijing Relativity Theory Research Federation, Shanghai Senior Scientist and technician Association. China.

2. Keming quantum Breeding Research Institute, Changsha, China.

\begin{abstract}
The Lorentz transformation derivation process of three different logics is shown and analyzed and compared. It is found that in the process of deriving the Lorentz transformation, the principle of the invariance of the speed of light can be used as a precondition, also can be a result of a logical reasoning. The different derivation methods of the Lorentz transformation will show different logical structures, giving different revelations, but they have intrinsic consistency, instant space-time's uniformity and symmetry.
\end{abstract}

Keywords: Lorentz transformation, Logical structure, Cosmological principle, Space-time theory.

\section{1、 Introduction}

Lorentz transformation has a variety of derivable methods [1,2], and different methods would show different logical structures. In this paper, the logic structure of three methods is analyzed. First, Einstein's derivation based on the two principle of the theory of relativity; second, the axiomatic method of symmetry between time and space; third, the derivation method of fluid mechanics. The latter two methods are proposed by the authors, and they do not need to preset the principle of light speed invariability.

The general derivation methods of Lorentz transformation proposed by Einstein mainly is to preset two principles, namely the principles of relativity and light speed invariability [3]. In fact, this derivation needs a third principle, the cosmological principle that space-time is homogeneous and isotropic [4,5]. Applying the cosmological principle can eliminate most of the undetermined coefficients, and the remaining undetermined coefficients can be solved by the principle of relativity and the invariability principle of light velocity. Here the logical structure of Lorentz transformation derivation is axiomatic system based on these three principles.

The axiomatic method of space-time symmetry requires only one axiom, the space-time's uniformity and the symmetry, the Lorentz transformation can be derived naturally. Here the speed of light is just a dimensional constant that can be coupled, and is not a logical premise. Therefore, the logical structure of this method is more concise than Einstein's. Both show the beauty of "logical physics".

The derivation method of fluid mechanics of Lorentz transform does not require any preset principle, so long as converting a compressible fluid into an incompressible fluid is substituted on the basis of Galileo transformation, the Lorentz transformation can be derived [6,7]. The derived method is very simple. It is not a pure logic of form, but a logic of matter with certain substantive links between things. Thus, it can guide us to reveal the physical mechanism of the theory of relativity.

Analyzing different logic structure deriving Lorentz transformation can make us to realize the significance of Lorentz transformation more comprehensively and objectively, and it is possible to lead us to find new growth points. 


\section{2、The general derivation methods of Lorentz transformation}

Einstein deduced the Lorentz transformation on the basis of relativity principle and the principle of light velocity invariability. It is the space-time transformation relationship between two inertial systems $S(x, y, z)$ and $S$ ' $^{\prime}\left(x^{\prime}, y^{\prime}, z^{\prime}\right)$. Firstly, according to the uniformity of space-time, this transformation must be linear, and its most general expression is:

$$
\left\{\begin{array}{l}
x^{\prime}=a_{11} x+a_{12} y+a_{13} z+a_{14} t \\
y^{\prime}=a_{21} x+a_{22} y+a_{23} z+a_{24} t \\
z^{\prime}=a_{31} x+a_{32} y+a_{33} z+a_{34} t \\
t^{\prime}=a_{41} x+a_{42} y+a_{43} z+a_{44} t
\end{array}\right.
$$

There are 16 undetermined coefficients in Eq. (1) . In order to reduce the undetermined coefficients, according to the uniformity and isotropic of space-time, the relative velocity between two inertial systems $\mathrm{S}(\mathrm{x}, \mathrm{y}, \mathrm{z})$ and $\mathrm{S}$ ' $\left(\mathrm{x}^{\prime}, \mathrm{y}^{\prime}, \mathrm{z}^{\prime}\right)$ can be selected along the common axis, and the corresponding coordinate plane is kept parallel, the origin $O=O^{\prime}, t=t^{\prime}=0$. Since these two inertial systems have no relative motion in the direction of $\mathrm{y}$ and $\mathrm{z}$ axes, therefore $a_{22}=a_{33}=1$, $a_{21}=a_{23}=a_{24}=a_{31}=a_{32}=a_{34}=0$, and $a_{42}=a_{43}=0$. When $x=0, x^{\prime}+v t^{\prime}=0$. So it is doable that let $x=\gamma\left(x^{\prime}+v t^{\prime}\right)$, where the $\gamma$ is a constant.

According to the principle of relativity, the form of physical equations should be the same in different inertial systems. Therefore, the above-mentioned event coordinates transformation from S-to-S' system is $x^{\prime}=\gamma(x-v t)$. And thus the Eq. (1) can be simplified as:

$$
\left\{\begin{array}{l}
x^{\prime}=a_{11}(x-v t) \\
y^{\prime}=y \\
z^{\prime}=z \\
t^{\prime}=a_{41} x+a_{44} t
\end{array}\right.
$$

There are only three unknowns in the Eq. (2), and if there are three equations, the problem will be solved. Here there is need the principle of light velocity invariability.

Let a spherical electromagnetic wave is emitted from the origin when $\mathrm{t}=0$, because the origins of the two inertial system coincidence, then, in these two inertial systems, electromagnetic spherical radius will be expanding with same rate, that is to say, there are two identical equation:

$$
\begin{aligned}
& x^{2}+y^{2}+z^{2}=c^{2} t^{2} \\
& x^{\prime 2}+y^{\prime 2}+z^{\prime 2}=c^{2} t^{\prime 2}
\end{aligned}
$$

Substituting (2) into (4) and comparing corresponding coefficients with the (3), so that the three equations can be obtained:

$$
\left\{\begin{array}{l}
c^{2} a_{44}^{2}-v^{2} a_{11}^{2}=c^{2} \\
a_{11}^{2}-c^{2} a_{41}^{2}=1 \\
v a_{11}^{2}+c^{2} a_{41}=0
\end{array}\right.
$$

So three unknown coefficients can be sought out: 


$$
\left\{\begin{array}{l}
a_{11}=1 / \sqrt{1-v^{2} / c^{2}} \\
a_{41}=-v / c^{2} \sqrt{1-v^{2} / c^{2}} \\
a_{44}=1 / \sqrt{1-v^{2} / c^{2}}
\end{array}\right.
$$

The result is the Lorenz transformation.

$$
\left\{\begin{array}{l}
x^{\prime}=\beta(x-v t) \\
y^{\prime}=y \\
z^{\prime}=z \\
t^{\prime}=\beta\left(t-\frac{v x}{c^{2}}\right)
\end{array} \quad\left(\beta=\frac{1}{\sqrt{1-v^{2} / c^{2}}}\right)\right.
$$

It can be seen from the above that, in this derivation, the principles of cosmology, relativity and light speed invariability are the presupposition. These principles are like the axioms in geometry, and the theory of relativity is a system of axioms. The axioms are the starting point or basis of logical reasoning, it cannot be proved in this system, that is to say, relativity cannot answer the question of why the speed of light is constant, and therefore it is difficult to explore the physical mechanism of relativity.

\section{3、The axiomatic method of space-time symmetry}

It is generally believed that the relativistic axiom system is quite simple and beautiful. Zhi-Heng Shi discovered: relativistic axiom system can be further simplified, deriving Lorentz transformation only requires a symmetry axiom of space-time. The axiom of symmetry is that space and time are not only homogeneous respectively, but they are symmetrical between them. Here, the principle of the light speed invariability does not need to be preset, and the speed of light would occurring naturally with the form of a coupling coefficient.

Let's look at the Galileo transformation. The Galileo transformation between the relatively static frame of reference $S_{1}\left(x_{1}, y_{1}, z_{1}\right)$ and the relatively moving frame of reference is:

$$
x_{2}=x_{1}-v t_{1} \quad y_{2}=y_{1} \quad z_{2}=z_{1} \quad t_{2}=t_{1}
$$

Obviously, the time and space in (8) is asymmetrical. Now, we assume that time and space are symmetric and homogeneous, and in this symmetric space-time, there is a relationship:

$$
x=\gamma(v)\left(x^{\prime}-v t^{\prime}\right) \quad y=y^{\prime} \quad z=z^{\prime} \quad t=\gamma(v)\left(t^{\prime}-a v x^{\prime}\right)
$$

Where the $a$ is the constant coefficient. The dimension of $a v x^{\prime}$ is time, $a \frac{L}{T} L=T$, therefore $a=T^{2} / L^{2}=1 / V^{2}$, the $V$ is a velocity dimension constant. According to the symmetry axiom of space-time, exchanging the moving and static frame of reference, the symmetry should be invariable, gives Eq. (10): 


$$
x^{\prime}=\gamma(-v)(x+v t) \quad y^{\prime}=y \quad z^{\prime}=z \quad t^{\prime}=\gamma(-\mathrm{v})(t+a v)
$$

The symmetry axiom of space-time require dimensionless factor $\gamma(v)=\gamma(-v)$. Substituting it into (9) and (10), solving functional equations, which can be obtained:

$$
\gamma(v)=1 / \sqrt{1-(a v)^{2}}=1 / \sqrt{1-v^{2} / V^{2}}
$$

The $\gamma(v)$ should be a real number, so $1-\mathrm{v}^{2} / V^{2} \geq 0, v \leq V$, which means that the $V$ is a speed that can't be exceeded by any object. So far this speed can only be the speed of light. Moreover, the experiments and Maxwell's equations also confirm or show that the speed of light is a biggest speed and invariable.

Substituting (11) into (9), it is just a Lorentz transformation.

Here, the precondition is very simple, and the principle of light velocity invariability is not a precondition, but a coupling coefficient which occur natural.

\section{4、 Deriving the Lorentz transformation with means of fluid}

\section{mechanics}

As early as in the 1980s, Changwei Hu (pen name Yuegong) pointed out that the Lorentz transformation can be derived by the method of fluid mechanics [8].

In the fluid mechanics, the velocity potential $\phi$ of an incompressible fluid satisfies the following equation:

$$
\Delta \phi(x . y . z)=0 .
$$

Let a body moves with velocity $v$ in an infinite compressible fluid, which causes disturbances in the velocity, density and pressure. If the disturbances are assumed to be infinitesimal quantities of the first order, the equation of linearization can be obtained[9]:

$$
\left(1-\frac{v^{2}}{c^{2}}\right) \frac{\partial^{2} \phi}{\partial x^{2}}+\frac{\partial^{2} \phi}{\partial y^{2}}+\frac{\partial^{2} \phi}{\partial z^{2}}=0 \quad\left(\frac{v}{c}<1\right),
$$

Where $c$ is the speed of sound in a fluids. The following transformation (14) can be used:

$$
\left\{\begin{array}{l}
x^{\prime}=\beta x \\
y^{\prime}=y \\
z^{\prime}=z
\end{array} \quad\left(\beta=\frac{1}{\sqrt{1-v^{2} / c^{2}}}\right)\right.
$$

Substituting (14) into (13), the resulting equation is identified with (1): $\Delta \phi\left(x^{\prime} \cdot y^{\prime} \cdot z^{\prime}\right)=0$.

Therefore, Eq. (14) is the transformation of the fluid from a compressible to an incompressible state. 
If two parallel right-angled coordinate systems $\mathrm{O}_{1}\left(x_{1}, y_{1}, z_{1}\right), \mathrm{O}_{2}\left(x_{2}, y_{2}, z_{2}\right)$ are constructed on two special fluids that satisfy equation (13), if their x-axes are superposed, and if $\mathrm{O}_{2}$ with speed $v$ moves in the positive $\mathrm{x}$-direction, a Galilean transformation can be performed between them, as in Eqs. (15) and (16):

$$
\begin{aligned}
& x_{2}=x_{1}-v t_{1}, y_{2}=y_{1}, z_{2}=z_{1} ; \\
& x_{1}=x_{2}+v t_{2}, y_{1}=y_{2}, z_{1}=z_{2} .
\end{aligned}
$$

(Note: here, the time $\mathrm{t}$ is written as $t_{1}$ and $t_{2}$ separately.)

Substituting (14) into (15) and (16), where $x_{1}$ in (15) and $x_{2}$ in (16) do not change because they are of the proper length, gives Eqs. (17) and (18):

$$
\begin{aligned}
& x_{2}^{\prime}=\beta\left(x_{1}^{\prime}-v t_{1}\right), y_{2}^{\prime}=y_{1}^{\prime}, z_{2}^{\prime}=z_{1}^{\prime} ; \\
& x_{1}^{\prime}=\beta\left(x_{2}^{\prime}+v t_{2}\right), y_{1}^{\prime}=y_{2}^{\prime}, z_{1}^{\prime}=z_{2}^{\prime} .
\end{aligned}
$$

Substituting the first equation in Eq. (17) into Eq. (18) leads to

$$
\begin{aligned}
t_{2}=\frac{1}{v \beta}\left(x_{1}^{\prime}-\beta^{2} x_{1}^{\prime}+\beta^{2} v t_{1}\right) & =\beta\left(t_{1}-\frac{x_{1}^{\prime}\left(\beta^{2}-1\right)}{v \beta^{2}}\right), \quad \beta^{2}=\frac{c^{2}}{c^{2}-v^{2}}, \\
t_{2} & =\beta\left(t_{1}-\frac{v x_{1}^{\prime}}{c^{2}}\right) .
\end{aligned}
$$

If the speed of sound $c$ in the special fluid is the speed of light in a vacuum, then the combination of (17) and (19) is the Lorentz transformation.

Above special fluid, whose distribution is infinite in space where the speed of sound is the speed of light in vacuum, cannot be a conventional fluid. It is, in fact, the physical vacuum, which was called Ether by us $[6,7]$.

Because the Galilean transformation expresses absolute time-space theory and the Lorentz transformation expresses relativistic space-time theory, therefore it has a clear physical meaning transforming compressibility ether in absolute time-space into incompressible ether in the four dimensional space-time, and thus, its derivation process is not pure logic of form, but the logic of matter with certain material significance.

The derivation of fluid mechanics of Lorenz transformation does not require any principle of predication. However, the ether in four-dimensional space-time is incompressible, which means that the distribution of ether density in relativistic space-time is uniform, and thus, the speed of light is constant of course.

\section{5、 Duscussion}

There seem great difference the three logic structures deriving Lorentz transformation, but they have something in common: the space and time are homogeneous before and after the transformation. The space and time were asymmetrical before the transformation, but they are 
symmetric after the transformation. Here's a question: is the time and space before and after transformation the one time and space? It is generally considered to be the one, the space-time theory expressed by the Galileo transformation is an approximate description; and the relativistic space-time theory is an accurate description. Such a view is wrong, in fact, they are two different space-time theories in nature. Absolute space-time is a real space-time, its uniformity is the absolute invariability of the space-time standard, while relativistic space-time is a material space-time, whose uniformity is the uniformity of the ether distribution in the four-dimensional space-time. The variability of relativistic space-time standard shows that relativistic space-time uniformity is an appearance in form, not an essence.

In the above three derived methods, the factors realized relativistic space-time uniformity, is the invariability principle of the light speed in Einstein's derived method; is the symmetry between space and time, and their uniformity in the axiomatic method of space-time symmetry; is the incompressibility of ether In the fluid mechanics method.

The invariability principle of the light speed is not necessary in the methods of time and space symmetry and fluid mechanics. Where, the light velocity, one is a constant that can be coupled; another is the sound of a fluid. Do it whether mean that the speed of light in the Lorentz transformation can be replaced by other parameters? Yes, it is so theoretically.

We have pointed out that relativity has certain limitations, only in its applicable range, the light speed is quantitatively the maximum. Beyond this range, there will be a new system of quantitative descriptions. However, so far the range of human understanding is all within the range of the application of relativity. The criteria of time, length, etc. are defined by light, reflecting this viewpoint [10].

Zhi-Heng Shi considers that the axiomatic method of space-time symmetry and Einstein's method all can be called logical physical method, and it is possible that they will be further developed into logical physics, which can complementing with Russell's logic mathematics principles.

We proposed the theory of compressibility ether on the basis of deriving the Lorentz transformation with means of fluid mechanics [6,7]. It contains the "logic of matter", and thus, can describe the physical mechanism of relativity. It believes that people are observing the world through the ether. In a low-speed, macroscopic system, the lens of ether, whose influence is negligible, looks like a flat mirror. What Newtonian physics describes is the physical reality that is unaffected by any medium. In high speed and microscopic systems, the influence of ether becomes apparent, and thus relativistic and quantum effects occur. The relativistic effect is the "lens effect" of macroscopic ether, and quantum effect is the "lens effect" of microcosmic ether. Microscopic ether is like a microlens array, which leads to quantum transition and probability wave phenomena.

Relativistic space-time is the space-time distorted by ether. Quantitatively, however, the theory of relativity is consistent with experimental data, because the experimental process can't avoid the influence of ether. This suggests that accurate quantitative descriptions do not necessarily reflect the truth of facts [11].

\section{References}

[1] Xiao-Jun Wang, Hong Guan, The derivation of the Lorenz transformation, COLLEGE 
PHYSICS, 081998.

[2] Shu-Feng Li, Yu Liu, Shu Zheng, Xue-Chun Li, Discussion on the derivation of Lorenz transformation based on teaching, Physics and Engineering, S1 2014.

[3] Einstein, A. On The Electrodynamics of Moving Bodies, Annalen der Physik 17: 891-921(1905).

[4] Somajit Dey, Time isotropy, Lorentz transformation and inertial frames, Studies in History and Philosophy of Modern Physics, DOI:10.1016/j.shpsb.2018.01.003.

[5] Sheng-Qi Feng, Full and necessary conditions for Lorenz transformation, Physics and Engineering, 042010.

[6] Chang-Wei Hu, Derivation of the relativistic equations from classical continuum mechanics on the basis of a macroscopic vacuum, Physics Essays, Volume 27: Pages 375-379, 2014.

[7] Chang-Wei Hu, The theory of compressibility ether, Modern physics, 2017, 7(4): 112-133. https://doi.org/10.12677/mp.2017.74013。

[8] Yue Gong, The ether theory of interval field, Ptential Science, 4, pp39-40, 1989.

[9] Fung, Y. C. A First Course in Continuum Mechanics, Beijing, Tsinghua University Press, pp. 257-259(2005).

[10] Chang-Wei Hu, The Confusion of Modern Cosmology and the New Model, ScienceOpen Preprints, DOI: 10.14293/S2199-1006.1.SOR-.PPTYNQB.v1

[11] Chang-Wei Hu. On the Quantitative Effects. International Journal of Modern Physics and Application. Vol. 1, No. 3, pp. 38-42. 2014. 